\title{
Predicting breast cancer response to neoadjuvant chemotherapy based on tumor vascular features in needle biopsies
}

Terisse A. Brocato, ${ }^{1}$ Ursa Brown-Glaberman, ${ }^{2}$ Zhihui Wang, ${ }^{3,4}$ Reed C. Selwyn, ${ }^{5,6}$ Colin M. Wilson, ${ }^{6}$ Edward F. Wyckoff,7 Lesley C. Lomo, ${ }^{8}$ Jennifer L. Saline, ${ }^{6}$ Anupama Hooda-Nehra, ${ }^{9,10}$ Renata Pasqualini, ${ }^{9,11}$ Wadih Arap, ${ }^{9,10}$ C. Jeffrey Brinker, ${ }^{1,712,13}$ and Vittorio Cristini ${ }^{3,4,14}$

'Department of Chemical and Biological Engineering and Center for Biomedical Engineering, University of New Mexico, Albuquerque, New Mexico, USA. ${ }^{2}$ University of New Mexico Comprehensive Cancer Center, Albuquerque, New Mexico, USA. ${ }^{3}$ Mathematics in Medicine Program, Houston Methodist Research Institute, Houston, Texas, USA. ${ }^{4}$ Department of Imaging Physics, University of Texas MD Anderson Cancer Center, Houston, Texas, USA. ${ }^{5}$ Center for Neuroscience and Regenerative Medicine, Uniformed Services University of the Health Sciences, Bethesda, Maryland, USA. ${ }^{6}$ Department of Radiology, University of New Mexico, Albuquerque, New Mexico, USA. ${ }^{7}$ Center for Micro-Engineered Materials, University of New Mexico, Albuquerque, New Mexico, USA. ${ }^{8}$ Department of Pathology, University of Utah, Salt Lake City, Utah, USA. ${ }^{9}$ Rutgers Cancer Institute of New Jersey, Newark, New Jersey, USA. ${ }^{10}$ Division of Hematology/Oncology, Department of Medicine, Rutgers New Jersey Medical School, Newark, New Jersey, USA. "Division of Cancer Biology, Department of Radiation Oncology, Rutgers New Jersey Medical School, Newark, New Jersey, USA. ${ }^{2}$ Department of Molecular Genetics and Microbiology, University of New Mexico Health Sciences Center, Albuquerque, New Mexico, USA. ${ }^{13}$ Self-Assembled Materials Department, Sandia National Laboratories, Albuquerque, New Mexico, USA. ${ }^{14}$ Department of Nanomedicine, Methodist Hospital Research Institute, Houston, Texas, USA.

In clinical breast cancer intervention, selection of the optimal treatment protocol based on predictive biomarkers remains an elusive goal. Here, we present a modeling tool to predict the likelihood of breast cancer response to neoadjuvant chemotherapy using patient-specific tumor vasculature biomarkers. A semiautomated analysis was implemented and performed on 3990 histological images from 48 patients, with 10-208 images analyzed for each patient. We applied a histology-based mathematical model to 30 resected primary breast cancer tumors and then evaluated a cohort of 18 patients undergoing neoadjuvant chemotherapy, collecting pre- and posttreatment pathology specimens and MRI data. We found that core biopsy samples can be used with acceptable accuracy to determine histological parameters representative of the whole tissue region. Analysis of model histology parameters obtained from tumor vasculature measurements, specifically diffusion distance divided by the radius of the drug-delivering blood vessel $\left(L / r_{b}\right)$ and blood volume fraction (BVF), provides a statistically significant separation of patients obtaining a pathologic complete response (pCR) from those who do not. With this model, it is feasible to evaluate primary breast tumor vasculature biomarkers in a patient-specific manner, thereby allowing a precision approach to breast cancer treatment.

contributed equally to this work.

Conflict of interest: The authors have declared that no conflict of interest exists.

Copyright: () 2019 American Society for Clinical Investigation

Submitted: November 30, 2018 Accepted: February 26, 2019 Published: April 18, 2019.

Reference information: /Cl Insight. 2019;4(8):e126518. https://doi. org/10.1172/jci.insight.126518.

\section{Introduction}

In the United States, breast cancer is the most common cancer in females and is the second most common cause of cancer death in women (1). While major advances have been achieved in treatment of early-stage breast cancer, many women still die from metastatic disease. The use of neoadjuvant chemotherapy has recently emerged as a promising method to increase treatment efficacy in patients with early-stage breast cancer, with improved patient survival shown to be correlated with complete eradication of invasive tumor in the primary breast lesion and lymph nodes (pathologic complete response [pCR]) upon surgery following neoadjuvant chemotherapy. This has been demonstrated in multiple clinical trials, including National Surgical Adjuvant Breast and Bowel Project (NSABP) protocol B18, in which 1523 women with 
early-stage breast cancer were randomly assigned to preoperative versus postoperative anthracycline-based chemotherapy (2). There was no difference in disease-free survival (DFS) or overall survival (OS) between treatment groups at 5 years. However, in the 683 women who received neoadjuvant treatment, outcomes were significantly better in women who achieved pCR compared with those without pCR (5-year OS $87.2 \%$ vs. $76.9 \%-78.4 \%, P=0.06$; DFS $83.6 \%$ vs. $60.3 \%-71.7 \%, P=0.0004$ ) after neoadjuvant therapy (3). Response to chemotherapy and pCR rates are known to vary by breast cancer subtype and chemotherapy regimen. This caveat notwithstanding, $\mathrm{pCR}$ has been shown to be a suitable surrogate end point for survival in patients with high-risk ER-positive/HER2-negative (i.e., luminal B), HER2-positive (nonluminal), and triple-negative disease, though not for those with low-risk ER-positive/HER2-negative (luminal A) breast cancer (4). Data also support that women who have a response to neoadjuvant chemotherapy but do not achieve a pCR have improved long-term outcomes when compared with those who do not respond, by using response in the primary breast lesion as a surrogate for chemosensitivity. Mittendorf et al. described and validated a novel breast cancer staging system for assessing prognosis after neoadjuvant chemotherapy on the basis of pretreatment clinical stage (CS), posttreatment pathologic stage (PS), and estrogen receptor status and grade (EG), known as the CPS+EG score (5). The ability of the CPS+EG score to stratify outcome was confirmed in both internal and external cohorts, with a score of $\leq 2$ corresponding with a 5-year disease-specific survival (DSS) ranging from $88 \%$ to $96 \%$, while DSS was reduced, with a score of $\geq 3$, ranging from $72 \%$ to $88 \%$ (5).

Neoadjuvant cytotoxic chemotherapy with an anthracycline plus taxane-based regimen, recommended as a preferred regimen by the National Comprehensive Cancer Network, results in pCR in only a minor subset of patients $(6,7)$. For example, in SWOG 0012, 185 patients with locally advanced breast cancer were treated with standard doxorubicin (Adriamycin) plus cyclophosphamide (AC) given every 21 days for 5 cycles, followed by weekly paclitaxel (T) for 12 weeks (8). Overall pCR rate was $21 \%$. However, in patients with hormone receptor-negative (HR-negative) tumors, the pCR rate was $29 \%$ compared with $11 \%$ in patients with HR-positive tumors. Tumor-specific biomarkers for predicting response have been explored, including tumor-infiltrating lymphocytes (TILs). TILs correlate with improved outcomes in several cancer types, including colorectal, ovarian, esophageal, renal, lung, pancreatic, and breast cancer $(9,10)$. Specific to early-stage breast cancer (of particular interest for this work), the presence of TILs in diagnostic needle core biopsy was shown to be an independent predictor of response to neoadjuvant $c$ hemotherapy (11), as was TIL density (12). Unfortunately, our clinical ability to a priori predict pCR to neoadjuvant therapy in breast cancer patients remains limited at best. Therefore, in order to maximize the utility of the neoadjuvant therapy strategy in multiple breast cancer subtypes, there is a clinical unmet need for tools to identify patients that are likely to respond to neoadjuvant cytotoxic chemotherapy, thereby allowing a precision approach to cancer treatment.

Over the years, our group has proposed that the characteristics of the tumor vasculature might be a biologic predictor of response to chemotherapy. This mechanistic hypothesis has been examined in a series of modeling studies to evaluate the prediction of treatment outcomes based on chemotherapy drug diffusion and the physical properties of several tumor types (13-29). We and other investigators have proposed that diffusion barriers may prevent drugs from reaching malignant tumor cells, a functional mechanism that might partially underlie drug resistance (30). Our mathematical model for predicting tumor response to chemotherapy (denoted by $f_{\text {kill }}$, i.e., the fraction of tumor killed due to therapy) has been retrospectively validated in patients with colorectal cancer (CRC) with metastasis to the liver (13). In metastatic CRC, the model predicted tumor response to chemotherapy using 3 drug perfusion- and diffusion-related parameters: blood volume fraction (BVF) in the tumor, the radius of blood vessels $\left(r_{\mathrm{b}}\right)$, and the drug diffusion distance in tumor tissue $(L)$; such parameters were tumor- and patient-specific, and thus were measured on an individual basis.

In this work, we have reasoned that the microanatomic cancer environment and functional attributes of the tumor-associated vasculature might be a biologic predictor of response to neoadjuvant chemotherapy in the setting of human breast cancer. We set out to test, validate, and expand our predictive mathematical model by rigorously applying it to 3 prospective groups of human breast cancer patients through an integrated evaluation of histopathology and multiphase contrast-enhanced magnetic resonance imaging (MCE-MRI) data with a computer-assisted semiautomated software to enable rapid yet robust throughput that may be adapted to routine clinical imaging settings. 


\section{Results}

Needle core biopsy feasibility. An overview of our research protocol is shown in Figure 1. We sought to determine the feasibility of using diagnostic needle core biopsies to inform a mathematical model for prediction of $f_{\text {kill }}$ in women with infiltrating ductal adenocarcinoma of the breast receiving neoadjuvant anthracycline/ taxane-based combination cytotoxic chemotherapy. As an initial step toward this goal, histopathological analysis to obtain model parameters was performed retrospectively on whole tumors from a cohort of breast cancer patients ( $n=30$, termed cohort A) who underwent upfront either lumpectomy or mastectomy (primary surgery without prior systemic cytotoxic therapy). For cohort A, we chose to evaluate the spectrum of breast cancer, including ER/PR-positive, HER2-positive, and triple-negative breast cancer. There was no detectable differentiation between patient groups (HR-positive tumors, HER2-positive, and triple-negative breast cancer) in cohort A with regard to model parameters by ANOVA (Supplemental Figure 1; supplemental material available online with this article; https://doi.org/10.1172/jci.insight.126518DS1).

Model parameters obtained from whole tumors in cohort A patients were subsequently compared with a similar analysis of histopathologic samples from diagnostic needle core biopsies of a second cohort ( $n=18$, termed cohort B) of high-risk stage II and III HER2-negative breast cancer (i.e., triple-negative and high-risk ER-positive/HER2) treated with neoadjuvant anthracycline/taxane-based chemotherapy (Figure 2). We found that cohort A had a higher BVF than cohort B, presumably due to the whole tumor section analysis in cohort A relative to the limited core biopsy samples in cohort B. Due to shape alone, the tissue section from a whole tumor section provides a larger highly vascularized tissue region (perimeter of tumor) for cohort A when compared with the cylindrical shape of a core biopsy for cohort B. This analysis also indicated that vasculature characteristics must be measured on an individual basis in breast cancer. We then performed nonlinear regression by fitting the $f_{\text {kill }}$ model to one of the patient histological parameters (i.e., BVF) specific to the tumor vasculature of each patient to determine the best fit for $L / r_{\mathrm{b}}$ for the entire dataset. We found that the patient samples for both cohorts fell along the same regression line (Figure 2); see fitting results in the inset. A correlation analysis between $f_{\text {kill }}\left[\mathrm{BVF}_{\text {biopsy }},\left(L / r_{\mathrm{b}}\right)_{\text {biopsy }}\right]$ versus $f_{\text {kill }}\left[\mathrm{BVF}_{\text {biopsy }},\left(L / r_{\mathrm{b}}\right)_{\text {fitting }}\right]$ for cohort B resulted in $r=0.7042$. Hence, we concluded that needle core biopsy samples may indeed be used to reliably determine histopathological parameters representative of the whole tissue.

Separation between clinical outcomes by $L / r_{b}$. The CPS+EG score, used as a method to quantify response to neoadjuvant chemotherapy, ranges from 0 to 6 , with a CPS+EG score $\leq 2$ corresponding to a 5 -year DSS from $88 \%$ to $96 \%$ (5). Our model was unable to discriminate between responders and nonresponders in cohort B by using a CPS+EG score of $\leq 2$ to define response. However, analysis of histopathology measurements, specifically $L / r_{\mathrm{b}}$, has provided a statistically significant separation of patients achieving a pCR from those that do not $(P=0.0269)$ (Figure 3$)$. We note that the obtained accuracy cannot be fully ascertained due to the small sample size, but the feasibility of using the parameter $L / r_{\mathrm{b}}$ to separate patients can be observed and further examined in future larger trials. We also note that all of the patients achieving a pCR in cohort B had triple-negative breast cancer. A single patient was identified as a clear outlier, likely due to the sample's dense population of TILs (not shown), an independent predictor of response to neoadjuvant chemotherapy $(11,12)$. Similar dense TIL infiltrates were not identified in the other 17 patients in cohort B.

$M C E-M R I A U C$ association to histology $L / r_{b}$. To evaluate model parameters via MRI, an AUC map in the tumor region was used to estimate tumor blood perfusion from MCE-MRI data, as described by Pickles et al. (31). In order to obtain quantitative data from MCE-MRI, a region of interest (ROI) must first be defined; here, the hotspot of the tissue ROI (tumor or control tissue) was used to determine the maximum perfusion in that tissue region (see Supplemental Figure 7 for visualization of hotspot). The hotspot region of the tumor was normalized to tissue in the equivalent anatomical location at the mirrored location on the contralateral breast (which represents a normal tissue region); this normalized value was used for analysis shown in Figure 4. We however note that patients further subdivided into categories based on $\mathrm{pCR}$ and ER/PR status did not demonstrate any further separation with histological parameters (Supplemental Figure 6). This may be due to the relatively small sample size used in our analysis, and is planned to be further investigated in the future. Correlation between AUC as determined by MCE-MRI and $L / r_{\mathrm{b}}$ as calculated by diagnostic needle core biopsy is shown in Figure 4 . As described above, in cohort $\mathrm{B}, L / r_{\mathrm{b}}$ demonstrated a positive correlation with pCR (i.e., the larger the $L / r_{\mathrm{b}}$ value, the better the chance was to achieve a pCR; see Figure 3 ). Our current analysis is limited by small sample size, but these pilot results that suggest a potential correlation 
1. Apply math modeling to Cohort A to identify primary resected whole tumor parameters

- Cohort A: 30 patients with primary resected breast cancer (10 ER/PR+, 10 HER2+, $10 \mathrm{TN}$ )

- Measure tumor parameters: blood volume fraction BVF, blood vessel radius $r_{b}$, and diffusion distance $L$, and calculate $f_{\text {kill }}$ using Eq. 1

- Evaluate model parameters by ER, PR, and HER2 status

2. Apply math modeling to Cohort B core biopsy samples as a surrogate for whole tumor analysis

- Cohort B: 18 patients treated with anthracycline/taxane based neoadjuvant chemotherapy

- Measure tumor parameters from core biopsy samples: blood volume fraction BVF, blood vessel radius $r_{\mathrm{b}}$, and diffusion distance $L$, and calculate $f_{\text {kill }}$ using Eq. 1

- Obtain pre-treatment perfusion measurements by diffusion contrast MRI as a proxy of tumor parameters

3. Apply model

- Evaluate the post-neoadjuvant chemotherapy clinical response in Cohort B patients at the time of surgery, including CPS+EG clinical scores and pCR

- Correlate $f_{\text {kill }}$ with pCR and CPS+EG score

- Correlate MRI results with response and histology results

Figure 1. Research protocol.

between AUC and $L / r_{\mathrm{b}}$ are encouraging. AUC may be assessed without core biopsy samples, a potential benefit given the limited specimen size obtained at the time of diagnostic biopsy and the increasingly common acquisition of pretreatment breast MRI in women receiving neoadjuvant chemotherapy. We further compared model predictions from histopathology data only [i.e., $f_{\text {kill }}$ (histology)] with that from MRI data only [i.e., $\left.f_{\text {kill }}(\mathrm{MRI})\right]$ and observed a weak correlation between these two predictions (Supplemental Figure 5). To further determine whether there exists a statistically significant correlation between $f_{\text {kill }}$ (histology) and $f_{\text {kill }}(\mathrm{MRI})$, a larger data set beyond the scope of this initial report will be required in future prospective studies.

\section{Discussion}

We have demonstrated the feasibility of evaluating breast cancer vasculature in a patient-specific manner with a customized semiautomated analysis. The quantities $r_{\mathrm{b}}$, BVF, and $L$ are shown to be reliably predictive of tumor $f_{\text {kill }}$ when obtained from standard diagnostic needle core biopsy in patients with stage II-III breast cancer, particularly those with triple-negative breast cancer achieving a pCR. Application of this model for clinical use at the initial diagnostic stage may allow noninvasive prediction of outcome, whereby likelihood of $\mathrm{pCR}$ can be estimated early in the course of treatment, by using the flowchart in Figure 5. The pilot framework introduced here represents steps toward the design of a subsequent larger prospective trials with our mathematical model to potentially select neoadjuvant chemotherapy treatment based on predicted response, treating only those patients most likely to have a response with standard anthracycline/taxane-based chemotherapy, while referring those unlikely to respond to other standard-ofcare options (e.g., radiation therapy) or even investigational clinical trials.

Tumor vasculature is a chaotic labyrinth of malformed and destabilized blood vessels that are structurally and functionally impaired (32). Jain has argued that drug delivery to tumors could be enhanced through tumor blood vessel normalization and reduced interstitial fluid pressure induced by antiangiogenic therapy (33). Along those lines of reasoning, a high $L / r_{\mathrm{b}}$ value in patients achieving a pCR is likely 


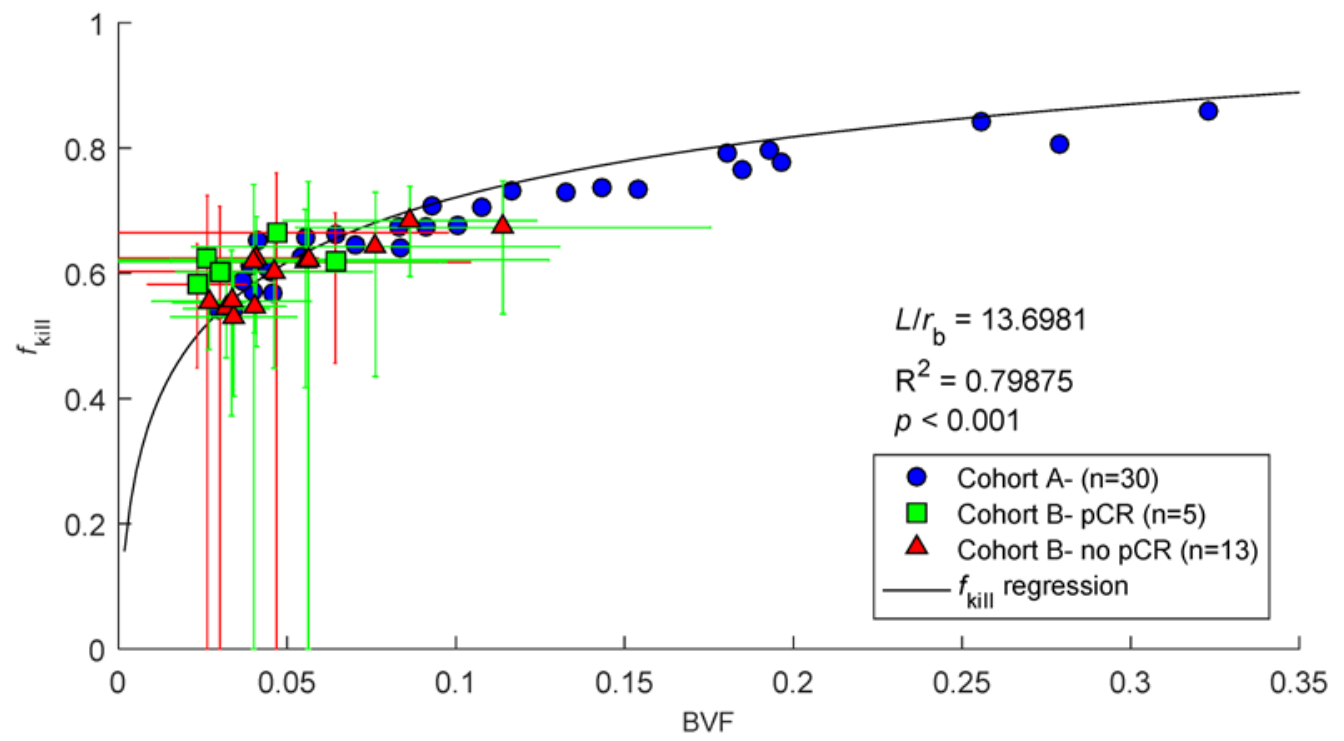

Figure 2. Model analysis. $f_{\text {kill }}$ values were determined as (i) calculated from measured values (points; cohort A: measured from resected whole-tumor histology, cohort B: measured from needle biopsy) and (ii) model best-fit (Equation 1) line to the full data set (black line). Cohort A: 30 patients underwent primary surgery without prior systemic therapy; data were analyzed by using histology semiautomated analysis and the mathematical model. Cohort B: 18 patients receiving neoadjuvant chemotherapy; data are shown to distinguish patients with pathologic complete response ( $\mathrm{pCR}$ ) versus those without $\mathrm{pCR}$. Each point is $f_{\text {kill }}$ calculated for an individual patient by using averages of $\mathrm{BVF}, r_{\mathrm{b}}$, and $L$ measured directly from tumor tissue stained with CD34 by immunohistochemistry. The black line shows $f_{\text {kill }}$ calculated from Equation 1 with optimized parameter $L / r_{\mathrm{b}}=13.6981$ (determined from fitting, $r^{2}=0.79875$ ). The $f_{\text {kill }}$ regression line includes fitting of both cohort $A$ and cohort $B$ patients $(n=48)$. Error bars are calculated based on error in BVF measurements and the respective variation that it causes when incorporated into the $f_{\text {kill }}$ equation (Equation 1). Correlation analysis of measured $f_{\text {kill }}$ and computed $f_{\text {kill }}$ for all 48 patients is shown in Supplemental Figure 2.

indicative of a more "normalized" baseline tumor vasculature, perhaps explaining improved response to chemotherapy in this subset of patients. A higher $L / r_{\mathrm{b}}$ value suggests that chemotherapy drugs may be more effectively delivered in these solid tumors, resulting in an improved kill fraction. Normal tissue has regularly spaced (or separated) blood vessels, which increases the value $L$ and thus also $L / r_{\mathrm{b}}$. Patient tumors with a high $L / r_{\mathrm{b}}$ values tended to have "pooled blood," or regions with highly vascularized tissue, severely limiting blood and drug delivery to poorly vascularized tumor regions. Model parameters correlated with $\mathrm{pCR}$ following neoadjuvant chemotherapy in women with triple-negative breast cancers, but they did not correlate with a less-than-complete response (i.e., CPS+EG score $\leq 2$ ) in triple-negative or high-risk ER-positive/HER2-negative breast cancers. We attribute this, in part, to our relatively small breast cancer patient population in the setting of a proof-of-concept study of a notoriously heterogeneous human tumor. Evaluation of our enabling mathematical platform in a larger breast cancer patient population might potentially allow the incorporation of other biologic features, including intensity of ER/PR expression, Ki-67 positivity, grade, and presence of TILs, to aid in predicting response to chemotherapy, particularly in those patients destined to achieve less than a $\mathrm{pCR}$.

Several technical aspects of the methodology merit further discussion. To begin, one of the limitations in our previous research in CRC (13) was that the histopathology parameters $L$ and $r_{\mathrm{b}}$ were solved for in the $f_{\text {kill }}$ model, while BVF was previously measured from H\&E-stained slides (13). Here, we have updated and refined this methodology by measuring these values $\left(L, r_{\mathrm{b}}\right.$, and BVF) directly from tissue sections, utilizing vasculature-specific staining to enable better visualization, increasing the accuracy of analysis. Measurements were previously done manually, which is both cumbersome and prone to human operator error. In contrast, a computer-assisted software program was customized here to allow for increased accuracy and speed in measurements; the semiautomated analysis allowed for rapid throughput, and a total of 3990 patient images were analyzed. Moreover, in this work we have correlated clinically relevant treatment response assessments ( $\mathrm{pCR}$ and CPS+EG score) with measured model parameters. Other limitations include differences in tumor vasculature staining via immunohistochemistry, although this source of bias has been greatly minimized 

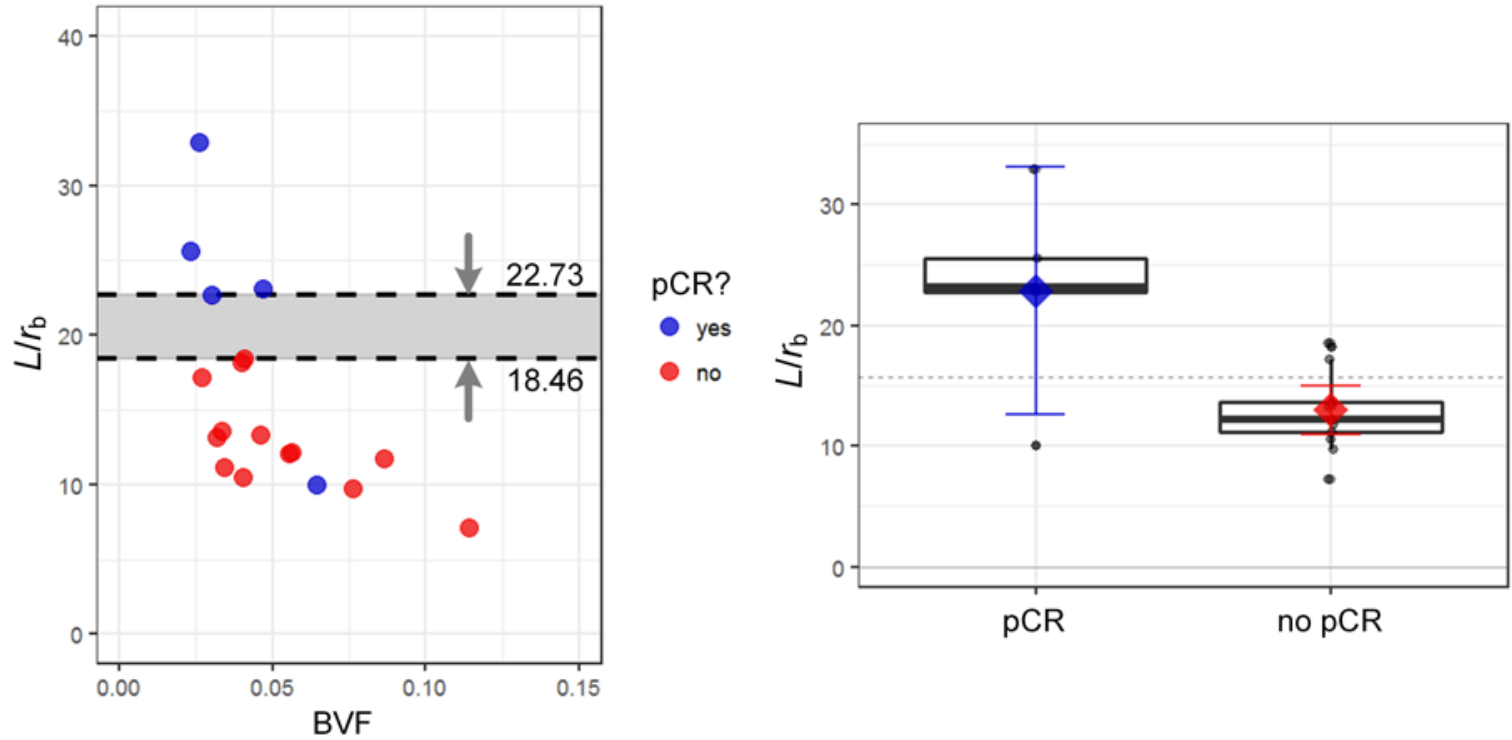

Figure 3. Histopathological parameters separate patient groups (pCR and no pCR). Left: Patient groups can be separated by a $L / r_{\mathrm{b}}$ value within the range of 18.46 (the highest value in the "no pCR" group) and 22.73 (the second-lowest value in the pCR group); see the gray zone. The patient from the pCR group that had the lowest $L / r_{b}$ value may be an outlier; see main text for details. Right: Student's $t$ test determined a statistically significant difference between the 2 groups with respect to $L / r_{b}(P<0.05)$. For each group, the box indicates the interquartile range and median, and the error bar denotes SD.

through an automated staining protocol. Finally, while the chemotherapy regimen used was internally consistent, we included all HER2-negative patients, resulting in a far more diverse patient population and thus increased heterogeneity in terms of response to neoadjuvant chemotherapy.

While we present exploratory data regarding the use of breast MRI to obtain model parameters, additional optimization is needed in future work. The clinical MRIs evaluated in this study were acquired with routine clinical protocols, which are focused on optimizing workflow and clinical radiology reporting instead of quantitative assessment for precision medicine. However, based on the pilot MRI data presented, AUC estimated from MCE-MRI analysis provided encouraging information regarding patient response. In our evaluation, hotspot ROI AUC analysis had the best correlation to treatment outcomes, when compared with looking at the whole tumor with 3D spherical ROI and a tumor ROI. Thus, the region perfused to the greatest extent seems to be the best predictor of treatment outcomes. There is a growing demand for and a body of evidence supporting development of precision imaging models. For the purposes of model parameter determination, MCE-MRIs should ideally contain a normalization method during acquisition to allow for a controlled method for T1 and B1 mapping (34). In order to obtain BVF from breast MRI, arterial input function might be evaluated at the time of scanning, with the ultimate inspirational goal of eliminating the need for needle core biopsy analysis for model prediction.

The semiautomated histology analysis described here can potentially be used for other solid tumors, although thresholding based on vascular staining and tumor types may need to be optimized for each. The general applicability of the mechanistic $f_{\text {kill }}$ model to predict response has been examined and confirmed in several other cancer types, including CRC with metastasis to liver, glioblastoma, pancreatic cancer, and lymphoma $(13,16,18,21)$. The observed consistency across tumor types is attributed to the fact that the $f_{\text {kill }}$ model was derived from fundamental principles of mass transport common to many solid tumor types (13) and evaluates vasculature characteristics in the tumor prior to treatment, thereby determining the efficiency of the vascular network to deliver drugs to the tumor. Our next steps will expand upon these results through inclusion of a large-scale data set containing more MRI measurements with additional time points, along with additional tumor parameters to predict response.

In summary, we report a mathematical modeling framework validated in patients with breast cancer from a single-institution study that is planned to be reproduced and further investigated in a large multi-institutional setting. If successful, the hypothesis-generating results introduced here may enable the future development of minimally invasive tools to accurately predict tumor response to neoadjuvant chemotherapy in patients with breast cancer. 


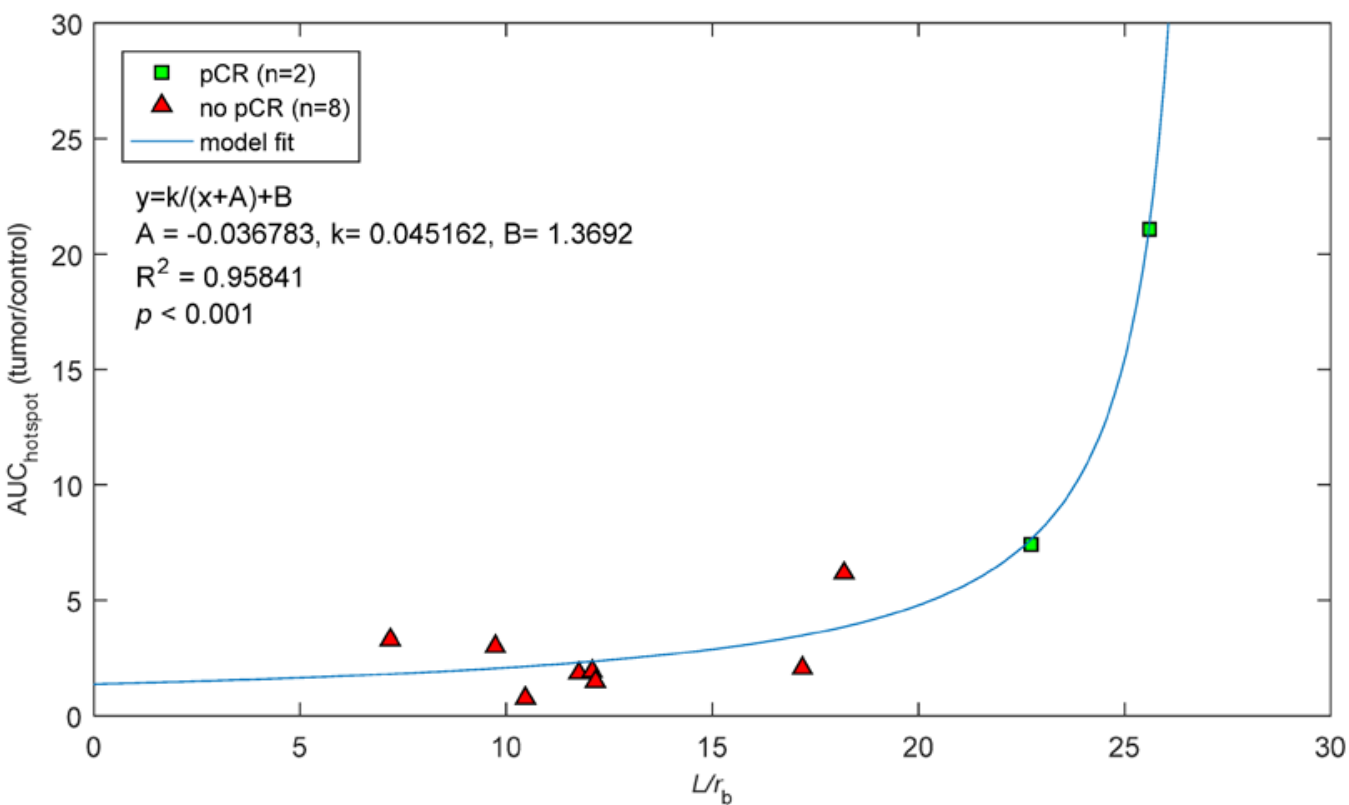

Figure 4. Response to neoadjuvant chemotherapy and MCE-MRI in cohort B. CD34-stained core biopsy samples measured for $L / r_{b}\left(r_{b}\right.$, radius of blood vessel; $L$, tissue diffusion penetration distance) and its relation to MCE-MRI AUC analysis, time points 0-5.5 minutes, taken for the hotspot region of the tumor normalized to the measured healthy tissue at the mirrored anatomical location on the contralateral breast.

\section{Methods}

Patient cohorts. Cohort A: The first step in evaluating our model in breast cancer was to analyze model parameters in primary resected breast tumors. We used primary resected tumors initially, as this provided ample tissue for histologic evaluation. In addition, we chose to evaluate a variety of breast cancer subtypes with regard to estrogen receptor, progesterone receptor, and HER2 expression, as it was unknown whether model parameters would vary by biomarker status. Thus, in cohort A, we retrospectively determined the parameters $r_{\mathrm{b}}, \mathrm{BVF}$, and $L$ from primary resected breast tumors, reviewing hormone (estrogen and progesterone) receptor-positive tumors $(n=10)$, HER2-positive tumors $(n=10)$, and tumors negative for both HRs and HER2 (triple-negative, $n=10$ ) utilizing de-identified archival paraffin-embedded tissue.

Cohort B: After determination of model parameters in cohort A, the model was applied to women who received neoadjuvant chemotherapy (cohort B). Cohort B $(n=18)$, as summarized in Table 1 , consisted of women with HER2-negative high-risk stage II-III infiltrating ductal carcinoma of the breast receiving neoadjuvant chemotherapy with a modern anthracycline/taxane-based regimen. High risk was based on stage and the opinion of the treatment provider (and tumor board) that neoadjuvant chemotherapy was warranted. As model parameters did not vary by biomarker status in cohort A, in cohort B we focused on exclusively HER2-negative patients to increase homogeneity with regard to chemotherapy regimen. In cohort B, paraffin-embedded baseline diagnostic needle core biopsy of primary breast tumor before chemotherapy was used to determine model parameters $r_{\mathrm{b}}$, BVF, and $L$ via semiautomated histopathology analysis as discussed below. In cohort A, we used whole tumor for analysis and found model parameters consistent across the tumor section despite tumor heterogeneity (as described below, identifying tumor, stroma, and vasculature). Thus, we felt confident moving to needle core biopsy alone for analysis in cohort B. In addition, in cohort B pre- and post-chemotherapy MRI, performed per standard of care, were used to obtain model parameters via an alternative imaging-based method (detailed below). Following neoadjuvant chemotherapy, all patients underwent surgical resection, allowing assessment of pathologic response and calculation of CPS+EG score.

Patient outcome evaluation. Patient treatment response was determined after completing neoadjuvant chemotherapy. Resected specimens were analyzed for pCR (yes/no). In addition, patient treatment response was assessed by using the CPS+EG score (5). Calculation of CPS+EG score was performed by the study team based on presenting clinical stage (obtained from pretreatment clinical notes), histologic 


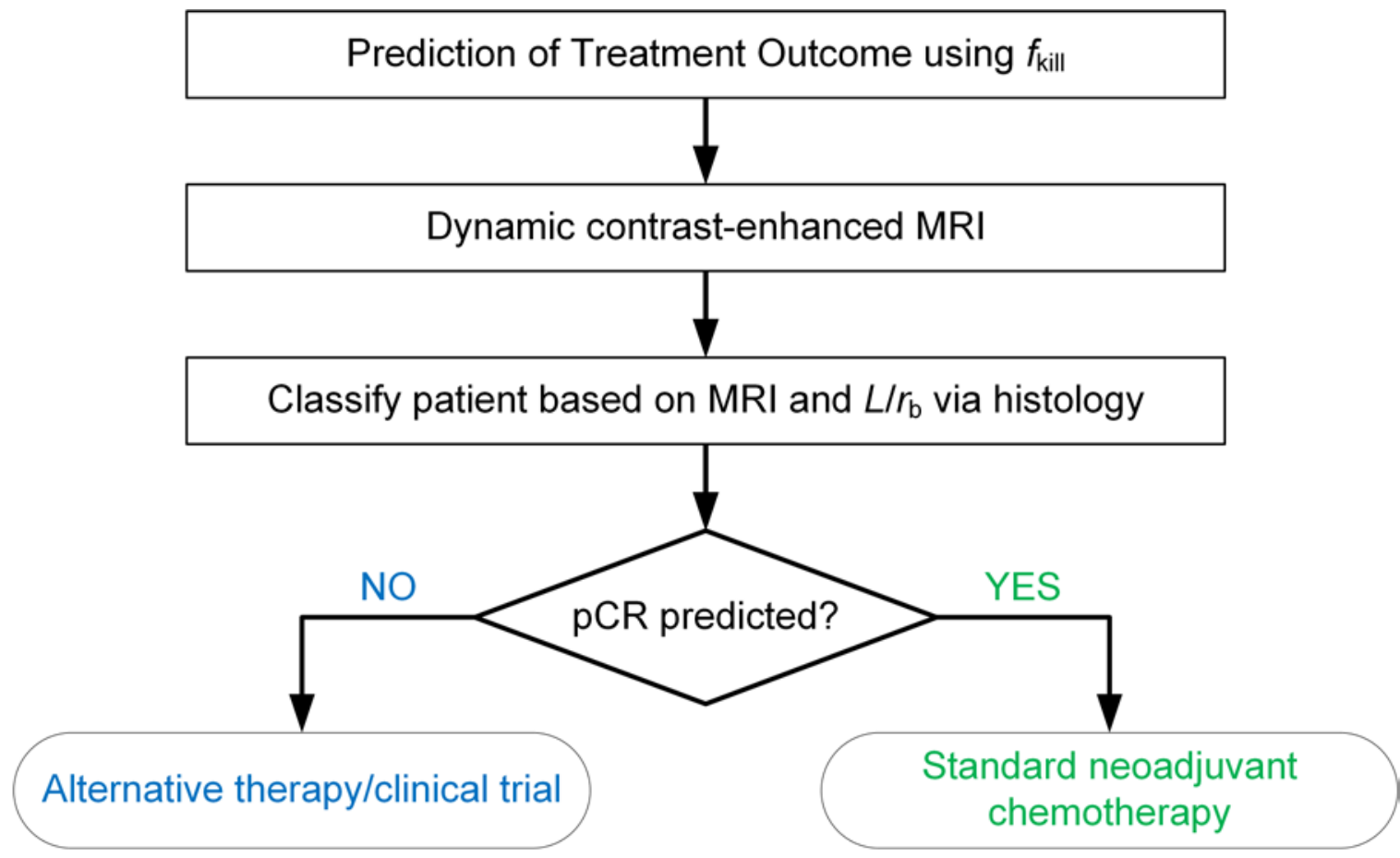

Figure 5. Prediction of treatment outcome using both MRI and tumor histology from diagnostic biopsies.

grade and estrogen receptor status (determined by routine pathology review of pre-treatment diagnostic biopsy), and post-neoadjuvant chemotherapy pathologic stage (determined by routine pathology review of post-treatment resection specimen) as described previously (5). Patient response was defined and analyzed by (i) pCR: no evidence of viable residual tumor in the primary resected breast specimen following the completion of neoadjuvant chemotherapy; and (ii) CPS+EG score $\leq 2$.

Magnetic resonance imaging. Patients had pretreatment and posttreatment gadolinium MCE-MRI scans on a 3T MRI (Siemens, Magnetom Tim Trio), which served as an imaging method to determine tumor and breast tissue perfusion. MRIs were obtained as part of routine clinical care prior to the administration of neoadjuvant chemotherapy (pretreatment) and after all planned neoadjuvant chemotherapy was administered prior to surgery (posttreatment). Baseline axial 3D gradient echo-based (FLASH) T1 scans were acquired without contrast using a dedicated 7 channel, receive-only breast coil with fat saturation and with the following parameters: 12-degree flip angle, 3.88/1.54 ms TR/TE, 0.9-mm slice thickness, $488 \times 358 \mathrm{FE} /$ $\mathrm{PE}$ matrix. Postcontrast images were acquired with the same parameters as baseline images with Magnevist $(0.2 \mathrm{ml} / \mathrm{kg}, 2 \mathrm{ml} / \mathrm{s})$ administered intravenously, with image acquisition at 1.5 minutes, 3.5 minutes, and 5.5 minutes after injection. Three subtraction images were created (post-pre contrast agent injection) and used for MRI analysis. Patients with MRIs not conforming to these criteria were excluded from the MRI analysis. Analysis of MRI data was performed with OsiriX Dynamic Contrast-Enhanced (DCE) Tool Plugin (35). AUC was measured by using a 3D spherical ROI over the tumor region determined by an attending radiologist, and the hotspot (maximum signal in a $1-\mathrm{cm}^{3}$ region given the original ROI) was measured for the tumor. For normalization, a control ROI was assessed on the contralateral breast in the same general anatomical position as the tumor, considered a baseline for the individual normal tissue vasculature in each patient. Supplemental Figure 7 shows representative MRI images along with the analyses performed.

Histopathology. Patient tissue samples were formalin fixed and paraffin embedded, and processed per institutional standard of care, in compliance with American Society of Clinical Oncology/College of American Pathology (ASCO-CAP) guidelines. The Human Tissue Repository and Tissue Analysis Shared Resource at the University of New Mexico Comprehensive Cancer Center (UNMCCC) served as an honest broker for access to all tumor specimens. CD34 antibody staining via immunohistochemistry was used to highlight tumor vasculature, and H\&E staining was performed to evaluate tissue morphology (e.g., tumor versus non-tumor). 
Table 1. Cohort B patient characteristics

\begin{tabular}{|c|c|}
\hline Patient/tumor parameter & $n(\%)$ \\
\hline HR-negative, HER2-negative & $9(50)$ \\
\hline HR-positive, HER2-negative & $9(50)$ \\
\hline \multicolumn{2}{|l|}{ Tumor grade } \\
\hline Grade 1 & $1(6)$ \\
\hline Grade 2 & $5(27)$ \\
\hline Grade 3 & $12(67)$ \\
\hline \multicolumn{2}{|l|}{ Clinical stage at presentation } \\
\hline Stage II & $12(67)$ \\
\hline Stage III & $6(33)$ \\
\hline \multicolumn{2}{|l|}{ Nodal status } \\
\hline Node positive & $11(61)$ \\
\hline Node negative & $7(39)$ \\
\hline \multicolumn{2}{|l|}{ Chemotherapy regimen } \\
\hline AC Q3 weeks $\times 4$ followed by T weekly $\times 12$ & $12(67)$ \\
\hline AC Q2 weeks × 4 (dd) followed by T weekly × 12 & $6(33)$ \\
\hline \multicolumn{2}{|l|}{ Response } \\
\hline $\mathrm{pCR}$ & $5(28)$ \\
\hline CPS + EG score $\leq 2$ & $10(56)$ \\
\hline
\end{tabular}
regions for analysis using code developed in MATLAB (MathWorks). This code takes the HALO-separated regions and measures vasculature radius, $r_{\mathrm{b}}(\mu \mathrm{m})$, along the short axis of the blood vessel due to the consideration that the blood vessel could be in the plane of the tissue section, thus ensuring we do not overestimate this parameter (Figure 6). Multiple measurements were taken for each blood vessel and averaged to obtain a single $r_{\mathrm{b}}$ value for each grid analyzed. To ensure the accuracy of the semiautomated methods developed, each measurement was checked multiple times over multiple iterations of the software analysis. BVF was taken to be the vasculature area (red, Figure 6C) divided by the whole tissue region (blue + red). Only tissue regions were considered for BVF measurement. Vessels were assumed to supply drug and nutrients to all surrounding tissue that was nearest to that vessel; these perimeters define the maximum diffusion length $L$ and are shown in black in Figure 6D. The distances between this boundary (black) and the nearest blood vessel (red) were measured and averaged for each grid analyzed to get the diffusion penetration distance $L$ $(\mu \mathrm{m})$. We note here that larger blood vessels (which presumably deliver greater amounts of drug) increase the $r_{\mathrm{b}}$ value, thus decreasing $L / r_{\mathrm{b}}$. This is balanced by $L$, which may have a longer penetration distance due to the increased vascular supply. In other words, it is the ratio of these two quantities that must be considered together instead of individually when evaluating treatment efficacy.

Mathematical model. Equation 1 shows the $f_{\text {kill }}$ equation with parameters $r_{\mathrm{b}}$, BVF, and $L$, which are directly measured from histology semiautomated analysis.

$f_{\text {kill }}=2 \cdot \operatorname{BVF} \cdot \frac{\sqrt{\mathrm{BVF}} \cdot K_{1}\left(r_{\mathrm{b}} / L\right)-K_{1}\left(r_{\mathrm{b}} /(L \cdot \sqrt{\mathrm{BVF}})\right)}{\sqrt{\mathrm{BVF}} \cdot r_{\mathrm{b}} / L \cdot K_{0}\left(r_{\mathrm{b}} / L\right) \cdot(1-\mathrm{BVF})}$

(Equation 1)

$f_{\text {kill }}$ is the fraction of tumor cells killed, $r_{\mathrm{b}}$ is the average radius of blood vessels in the tissue section analyzed, BVF is the fraction of blood volume in the tumor, and $L$ is the farthest distance nutrients/drug need to travel from a blood vessel to reach all tissue (13).

Statistics. MATLAB and GraphPad Prism 7 were used to determine best fits of patient averages for BVF, $r_{\mathrm{b}}$, and $L$ placed into Equation 1 and by using nonlinear regression solving for $L / r_{\mathrm{b}}$. Fits were obtained with initial values for fit, $L / r_{\mathrm{b}}=20$ and $L / r_{\mathrm{b}}>0.005$ for a constraint. For data in Figure 3, 2-tailed Student's $t$ test was used to compare histopathology measurements $\left(L / r_{\mathrm{b}}\right)$ from the 2 groups (pCR and no pCR). $P<0.05$ 
A
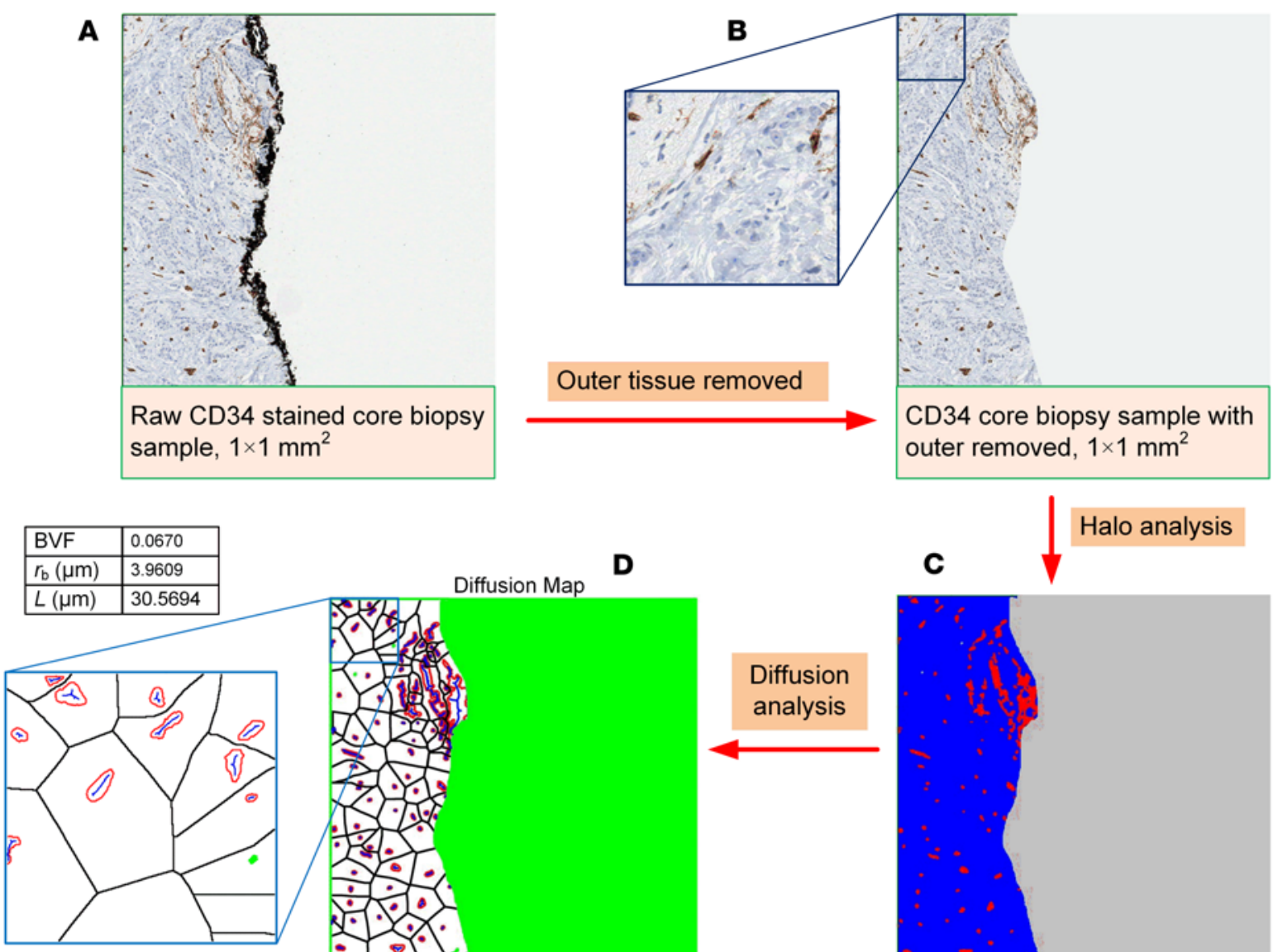

Halo Diffusion Analysis Map

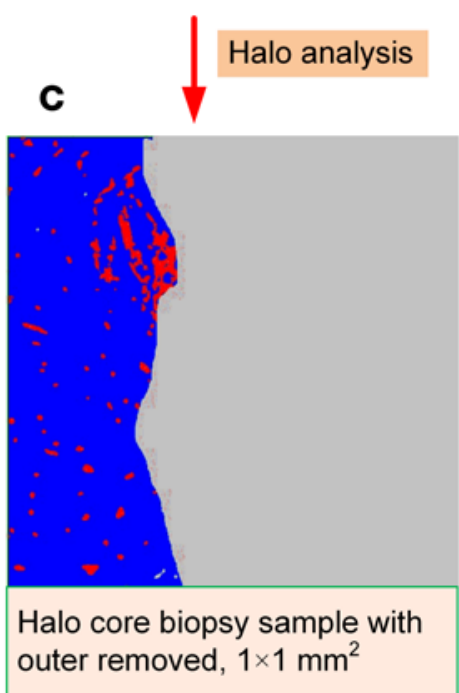

Figure 6. Diffusion analysis workflow. (A) Original CD34-stained histology grid before any processing. (B) Same tissue region as in A, but with the outer inked portion removed due to the increased likelihood of false positives on the perimeter of core biopsy samples (the University of New Mexico Pathology Department inks tissue cores for quality purposes). (C) Computerized version of B with differentiation between tissue CD34- (blue), vasculature CD34 (red), and non-tissue regions (gray). (D) Diffusion analysis of image in C, which was performed by code developed in MATLAB. Parameters measured were: blood vessel radius $\left(r_{b}\right)$, blood volume fraction (BVF), and diffusion distance $(L)$ ). Blood vessels are outlined in red, and the total area of blood vessels in a tissue region is the BVF. Blue shows the central long axis of each vessel (multiple vessel radius measurements were taken perpendicular to this axis). An average of all vessel radii in each image analyzed is taken to be $r_{\mathrm{b}}(\mu \mathrm{m})$. The black lines discretize the image into regions defined by having the closet proximity to the enclosed vessel; then the distance from each black boundary to the blood vessel boundary (red) is measured; and all distances averaged is the diffusion penetration distance $(L)$ measured in $\mu \mathrm{m}$. White is the tumor tissue region, all of which is considered for analysis. Green is the background/non-tissue region not considered for analysis.

was considered statistically significant. Additional analysis was done to compare BVF and $L / r_{\mathrm{b}}$ to determine whether one parameter can be omitted (see Supplemental Figure 3), and to determine whether $f_{\text {kill }}$ can be predicted with one-parameter BVF, which can be obtained from MRI (see Supplemental Figure 4).

Study approval. For all components of this research, approvals were obtained from the IRB of the University of New Mexico Health Sciences Center, study IDs 14-070 and 15-017. Studies were conducted according to the principles set out in the Declaration of Helsinki. Written informed consent was obtained from all prospective study patients.

\section{Author contributions}

TAB performed histopathology analysis, MRI analysis, and code development, and interpreted and analyzed the data. UBG, LCL, JLS, and VC initiated the project and designed the patient treatment protocol. UBG, LCL, and JLS performed cancer care and patient trial enrollment. UBG and LCL provided human tissues and supervised human histopathological data interpretation. ZW supervised data analysis, code 
development, and modeling. RGS and CMW participated in MRI analysis and interpretation. EFW provided assistance in histological data analysis and code development. AHN, RP, WA, and CJB participated in data analysis. ZW, RP, WA, and VC coordinated and supervised the overall research effort. TAB, UBG, $\mathrm{ZW}, \mathrm{RP}, \mathrm{WA}$, and VC wrote the manuscript.

\section{Acknowledgments}

We thank trial participants and study clinicians. We also give thanks to the Human Tissue Repository and Fred Schultz for his contribution in using the HALO system at the University of New Mexico. This research was supported by the National Cancer Institute and the Cancer Center Support Grant P30CA118100. This research has also been supported in part by a New Mexico Cancer Nanoscience and Microsystems Training Center (CNTC) Graduate Student Fellowship (TAB); National Science Foundation Grant DMS-1716737 (ZW, VC); NIH grants 1U01CA196403 (ZW, VC), 1U01CA213759 (ZW, VC), 1R01CA226537 (ZW, RP, WA, CJB, VC), 1R01CA222007 (ZW, VC), U54CA210181 (ZW, VC); and the University of Texas System STARS Award (VC). Sandia National Laboratories is a multi-mission laboratory managed and operated by National Technology and Engineering Solutions of Sandia LLC, a wholly owned subsidiary of Honeywell International Inc., for the US Department of Energy's National Nuclear Security Administration under contract DE-NA0003525. This manuscript describes objective technical results and analysis. Any subjective views or opinions that might be expressed in the paper do not necessarily represent the views of the US Department of Energy or the United States Government. The funders had no role in study design, data collection and analysis, decision to publish, or preparation of the manuscript.

Address correspondence to: Zhihui Wang, Mathematics in Medicine Program, The Houston Methodist Research Institute HMRI R8-122, 6670 Bertner Ave, Houston, Texas 77030, USA. Phone: 713.441.7291; Email: zwang@houstonmethodist.org. Or to: Wadih Arap, Rutgers Cancer Institute of New Jersey, Newark, New Jersey 07730, USA. Phone: 973.972.0366; Email: wadih.arap@rutgers.edu. Or to: Vittorio Cristini, Mathematics in Medicine Program, The Houston Methodist Research Institute, HMRI R8-123, 6670 Bertner Ave, Houston, Texas 77030, USA. Phone: 505.934.1813; Email: vcristini@houstonmethodist.org.

1. Miller KD, et al. Cancer treatment and survivorship statistics, 2016. CA Cancer J Clin. 2016;66(4):271-289.

2. Wolmark N, Wang J, Mamounas E, Bryant J, Fisher B. Preoperative chemotherapy in patients with operable breast cancer: nineyear results from National Surgical Adjuvant Breast and Bowel Project B-18. J Natl Cancer Inst Monogr. 2001;2001(30):96-102.

3. Fisher B, et al. Effect of preoperative chemotherapy on the outcome of women with operable breast cancer. JClin Oncol. 1998;16(8):2672-2685.

4. von Minckwitz G, et al. Definition and impact of pathologic complete response on prognosis after neoadjuvant chemotherapy in various intrinsic breast cancer subtypes. J Clin Oncol. 2012;30(15):1796-1804.

5. Mittendorf EA, et al. Validation of a novel staging system for disease-specific survival in patients with breast cancer treated with neoadjuvant chemotherapy. J Clin Oncol. 2011;29(15):1956-1962.

6. Mieog JS, van der Hage JA, van de Velde CJ. Preoperative chemotherapy for women with operable breast cancer. Cochrane Database Syst. 2007;(2):CD005002.

7. Mauri D, Pavlidis N, Ioannidis JP. Neoadjuvant versus adjuvant systemic treatment in breast cancer: a meta-analysis. $J$ Natl Cancer Inst. 2005;97(3):188-194.

8. Ellis GK, et al. Phase III comparison of standard doxorubicin and cyclophosphamide versus weekly doxorubicin and daily oral cyclophosphamide plus granulocyte colony-stimulating factor as neoadjuvant therapy for inflammatory and locally advanced breast cancer: SWOG 0012. J Clin Oncol. 2011;29(8):1014-1021.

9. Mahmoud SM, et al. Tumor-infiltrating CD8+ lymphocytes predict clinical outcome in breast cancer. J Clin Oncol. 2011;29(15):1949-1955.

10. Denkert C, et al. Tumor-infiltrating lymphocytes and response to neoadjuvant chemotherapy with or without carboplatin in human epidermal growth factor receptor 2-positive and triple-negative primary breast cancers. J Clin Oncol. 2015;33(9):983-991.

11. Denkert C, et al. Tumor-associated lymphocytes as an independent predictor of response to neoadjuvant chemotherapy in breast cancer. J Clin Oncol. 2010;28(1):105-113.

12. Ali HR, et al. Computational pathology of pre-treatment biopsies identifies lymphocyte density as a predictor of response to neoadjuvant chemotherapy in breast cancer. Breast Cancer Res. 2016;18(1):21.

13. Pascal J, Bearer EL, Wang Z, Koay EJ, Curley SA, Cristini V. Mechanistic patient-specific predictive correlation of tumor drug response with microenvironment and perfusion measurements. Proc Natl Acad Sci USA. 2013;110(35):14266-14271.

14. Das H, et al. Impact of diffusion barriers to small cytotoxic molecules on the efficacy of immunotherapy in breast cancer. PLoS ONE. 2013;8(4):e61398.

15. Pascal J, et al. Mechanistic modeling identifies drug-uptake history as predictor of tumor drug resistance and nano-carrier-mediated response. ACS Nano. 2013;7(12):11174-11182.

16. Koay EJ, et al. Transport properties of pancreatic cancer describe gemcitabine delivery and response. J Clin Invest. 2014;124(4):1525-1536. 
17. Brocato T, et al. Understanding drug resistance in breast cancer with mathematical oncology. Curr Breast Cancer Rep. 2014;6(2):110-120.

18. Frieboes HB, et al. Predictive modeling of drug response in non-hodgkin's lymphoma. PLoS One. 2015;10(6):e0129433.

19. Wang Z, Butner JD, Kerketta R, Cristini V, Deisboeck TS. Simulating cancer growth with multiscale agent-based modeling. Semin Cancer Biol. 2015;30:70-78.

20. Wang Z, Butner JD, Cristini V, Deisboeck TS. Integrated PK-PD and agent-based modeling in oncology. J Pharmacokinet Pharmacodyn. 2015;42(2):179-189.

21. Wang Z, et al. Theory and experimental validation of a spatio-temporal model of chemotherapy transport to enhance tumor cell kill. PLoS Comput Biol. 2016;12(6):e1004969.

22. Cristini, V., Koay, E., Wang, Z. An Introduction to Physical Oncology: How Mechanistic Mathematical Modeling Can Improve Cancer Therapy Outcomes. Boca Raton, Florida, USA: CRC Press; 2017.

23. Frieboes HB, Chaplain MA, Thompson AM, Bearer EL, Lowengrub JS, Cristini V. Physical oncology: a bench-to-bedside quantitative and predictive approach. Cancer Res. 2011;71(2):298-302.

24. Edgerton ME, Chuang YL, Macklin P, Yang W, Bearer EL, Cristini V. A novel, patient-specific mathematical pathology approach for assessment of surgical volume: application to ductal carcinoma in situ of the breast. Anal Cell Pathol (Amst). 2011;34(5):247-263.

25. Deisboeck TS, Wang Z, Macklin P, Cristini V. Multiscale cancer modeling. Annu Rev Biomed Eng. 2011;13:127-155.

26. Wang Z, Bordas V, Deisboeck TS. Identification of critical molecular components in a multiscale cancer model based on the integration of Monte Carlo, resampling, and ANOVA. Front Physiol. 2011;2:35.

27. Wang Z, Deisboeck TS, Cristini V. Development of a sampling-based global sensitivity analysis workflow for multiscale computational cancer models. IET Syst Biol. 2014;8(5):191-197.

28. Brocato TA, et al. Understanding the connection between nanoparticle uptake and cancer treatment efficacy using mathematical modeling. Sci Rep. 2018;8(1):7538.

29. Dogra P, et al. Establishing the effects of mesoporous silica nanoparticle properties on in vivo disposition using imaging-based pharmacokinetics. Nat Commun. 2018;9(1):4551.

30. Adiseshaiah PP, Crist RM, Hook SS, McNeil SE. Nanomedicine strategies to overcome the pathophysiological barriers of pancreatic cancer. Nat Rev Clin Oncol. 2016;13(12):750-765.

31. Pickles MD, Manton DJ, Lowry M, Turnbull LW. Prognostic value of pre-treatment DCE-MRI parameters in predicting disease free and overall survival for breast cancer patients undergoing neoadjuvant chemotherapy. Eur J Radiol. 2009;71(3):498-505.

32. Nagy JA, Chang SH, Dvorak AM, Dvorak HF. Why are tumour blood vessels abnormal and why is it important to know? Br J Cancer. 2009;100(6):865-869.

33. Jain RK. Normalization of tumor vasculature: an emerging concept in antiangiogenic therapy. Science. 2005;307(5706):58-62.

34. Pineda FD, Medved M, Fan X, Karczmar GS. B1 and T1 mapping of the breast with a reference tissue method. Magn Reson Med. 2016;75(4):1565-1573.

35. Sung K, Daniel B, Rubin D, Hargreaves B. Quantitative dynamic contrast-enhanced MRI analysis tool. Abstract presented at: Radiological Society of North America 2011 Scientific Assembly and Annual Meeting, November 26-December 2, 2011, Chicago Illinois, USA. http://archive.rsna.org/2011/11015719.html. Accessed February 27, 2019. 